\title{
A cluster randomised control trial to evaluate the effectiveness and cost- effectiveness of the Italian medicines use review (I-MUR) for asthma patients
}

\author{
Andrea Manfrin ${ }^{1 *}$, Michela Tinelli ${ }^{2}$, Trudy Thomas ${ }^{1}$ and Janet Krska ${ }^{1}$
}

\begin{abstract}
Background: The economic burden of asthma, which relates to the degree of control, is $€ 5$ billion annually in Italy. Pharmacists could help improve asthma control, reducing this burden. This study aimed to evaluate the effectiveness and cost-effectiveness of Medicines Use Reviews provided by community pharmacists in asthma.

Methods: This cluster randomised, multi-centre, controlled trial in adult patients with asthma was conducted in 15 of the 20 regions of Italy between September 2014 and July 2015. After stratification by region, community pharmacists were randomly allocated to group A (trained in and delivered the intervention at baseline) or B (training and delivery 3 months later), using computerised random number generation in blocks of 10. Each recruited up to five patients, with both groups followed for 9 months.

The intervention consisted of a systematic, structured face-to-face consultation with a pharmacist, covering asthma symptoms, medicines used, attitude towards medicines and adherence, recording pharmacist-identified pharmaceutical care issues ( $\mathrm{PCl}$ ). The primary outcome was asthma control, assessed using the Asthma-Control-Test (ACT) score (ACT $\geq 20$ represents good control). Secondary outcomes were: number of active ingredients, adherence, cost-effectiveness compared with usual care. Although blinding was not possible for either pharmacists or patients, assessment of outcomes was conducted by researchers blind to group allocation.

Results: Numbers of pharmacists and patients enrolled were $283(A=136 ; B=147)$ and $1263(A=600 ; B=663)$, numbers completing were $201(A=97 ; B=104)$ and $816(A=400 ; B=416)$, respectively. Patients were similar in age and gender and 56.13\% (458/816) had poor/partial asthma control. Pharmacists identified $1256 \mathrm{PCls}$ (mean 1.54/patient), mostly need for education, monitoring and potentially ineffective therapy. Median ACT score at baseline differed between groups $(A=19, B=18 ; p<0.01)$. Odds ratio for improved asthma control was 1.76 (95\% Cl 1.33-2.33) and number needed to treat 10 (95\% Cl 6-28). Number of active ingredients reduced by $7.9 \%$ post-intervention $(p<0.01)$. Adherence improved by $35.4 \% 3$ months post-intervention and $40.0 \%$ at 6 months $(p<0.01)$. The probability of the intervention being more cost-effective than usual care was $100 \%$ at 9 months.
\end{abstract}

Conclusions: This community pharmacist-based intervention demonstrated both effectiveness and costeffectiveness. It has since been implemented as the first community pharmacy cognitive service in Italy.

Trial registration: TRN: ISRCTN72438848 (registered $5^{\text {th }}$ January 2015, retrospectively).

Keywords: Asthma control, Medicines use review, Community pharmacy, Effectiveness, Cost-effectiveness

\footnotetext{
* Correspondence: a.manfrin@kent.ac.uk

${ }^{1}$ Medway School of Pharmacy, Universities of Greenwich and Kent at

Medway, Anson Building, Central Avenue, Chatham Maritime, Chatham, Kent

ME4 4TB, UK

Full list of author information is available at the end of the article
} 


\section{Background}

The prevalence of asthma has been increasing since the late 1990's and it has been estimated that about 400 million people will suffer from asthma by $2025[1,2]$. Currently the number of disability-adjusted life years (DALYs) lost to lack of control of asthma worldwide translates into a global loss of 15 million DALYs per year and an estimated one in every 250 deaths worldwide is caused by asthma. Asthma accounts for an economic loss of $€ 72$ billion annually in the 28 countries of the EU [3]; this includes the annual costs of health care (about $€ 20$ billion), the loss of productivity for patients ( $€ 14$ billion), and a monetised value of DALYs lost of $€ 38$ billion. In the UK the burden of asthma was estimated at a cost to the NHS of more than $£ 1$ billion/year, including prescribing, hospital admissions and primary care consultations $[4,5]$. In Italy the latest estimate showed an asthma prevalence of $6.2 \%$ [6] with an economic loss of $€ 5$ billion annually. An Italian cost of illness study, published in 2000, reported a mean annual cost for an asthmatic patient of $€ 741$ (95\% CI: 599-884), ranging from $€ 379$ (95\% CI: 216-541) for well-controlled asthmatics to $€ 1,341$ (95\% CI: 978-1,706) for poorly controlled cases, the latter accounting for $46.2 \%$ of the total cost of asthma [7]. In 2006 the Global Initiative for Asthma (GINA) advocated a new asthma management approach, based on asthma control rather than asthma severity [8], since "the principal objective of asthma treatment is to achieve asthma control". The GINA 2015 pocket guide for asthma management and prevention [9] defines asthma control as the extent to which the effects of asthma can be seen in patients, or has been reduced or removed by treatment. While guidance on asthma control abounds in the UK $[10,11]$ and other countries, [12] many patients do not achieve good asthma control, with negative implications for their health and quality of life, as well as increased health care costs and loss of productivity $[6,13,14]$. Asthma control may be affected by co-morbidity, continued exposure to triggers, behavioural issues, patient preferences, ineffective treatment, poor adherence and costs for patients [15].

Pharmaceutical care, defined in Europe [16] as "the pharmacists' contribution to the care of individuals in order to optimise medicines use and improve health outcomes", could contribute to asthma management. Many studies report positive effects of pharmacist interventions in asthma [17-28]. Indeed, a systematic review published in 2016 [29] looking at the impact of pharmacists' intervention on clinical outcomes in asthma identified 21 studies. However of these, only nine adopted a randomised control or a cluster randomised control design, only ten measured asthma control as main outcome as suggested by GINA management approach in assessing effectiveness, [9] and only one included an economic evaluation of the pharmacy intervention [22]. Pharmacy-led approaches to asthma management include both one-off interventions, such as Medicines Use Review (MUR), and longer-term provision of pharmaceutical care. In England, the MUR is a cognitive pharmaceutical service, funded by the government, [30] where accredited pharmacists can undertake structured adherence-centred reviews with patients using multiple medicines, particularly those receiving medicines for asthma and other longterm conditions. Similar services exist in some European (Denmark, Switzerland) and other countries (Australia, Canada, New Zealand, USA) [31], whereas in Italy such services (for asthma or other conditions) have not been introduced. Therefore, no empirical research exists addressing the effectiveness or cost-effectiveness of a MUR service in Italy.

This study describes the first attempt to deliver a novel community pharmacy intervention for asthma patients, adapted from the MUR service for chronic diseases in England, across the Italian territory and to evaluate its effectiveness and cost-effectiveness. The key research questions were:

Is the Italian Medicines Use Review (I-MUR) service provided by community pharmacists:

\section{i. Effective in}

a. Improving asthma control as assessed by the Asthma Control Test (ACT) score?

b. Optimising the number of medicines (active ingredients) used by patients?

c. Identifying pharmaceutical care issues?

d. Improving patients' adherence to asthma medications?

ii. Cost-effective for the healthcare system and the society (compared with usual care) in terms of cost per quality adjusted life year gained?

\section{Methods}

The study was a cluster randomised controlled trial, with phased intervention, as defined by Gums et al. (2016) [32], in order to avoid selection bias, with control clusters providing the intervention after the primary end point (primary outcome) was finished (T3). Therefore all pharmacists (intervention and control) provided the I-MUR service to patients with asthma, but at different time points during the study. The study protocol is published elsewhere [33].

Community pharmacists were recruited from 15 out of the 20 Italian regions: Trentino Alto Adige, Lombardia, Sicilia, Puglia, Sardegna, Piemonte, Valle d'Aosta, Veneto, Friuli Venezia Giulia, Toscana, Emilia Romagna, Marche, Abruzzo, Lazio and Campania. It was conducted between September 2014 and July 2015 with data collection at 3-months intervals (at baseline (T0); at 3 months (T3); at 6 months (T6); at 9 months (T9)). Pharmacists were randomly allocated to two groups after stratification by region: group A were trained to provide the I-MUR 
immediately after completion of the baseline ACT score at T0; group $\mathrm{B}$ received training and provided this service 3 months later, at $\mathrm{T} 3$.

\section{Intervention}

The I-MUR consisted of a systematic, structured interview, conducted in a private room within the pharmacy, which covered asthma symptoms, medicines used, attitudes towards medicines and adherence. The pharmacists were trained to identify pharmaceutical care issues (PCIs) which could impact on optimal medicines use or asthma control and provide advice to the patients and recommendations to their GP, as necessary. As a process measure, the number of PCIs identified by pharmacists during the I-MUR service provision, classified using the method of Krska et al [34], were recorded.

\section{Outcome measures}

Primary outcome measure: Asthma control at baseline (T0) and at 3-months intervals (T3, T6 and T9), assessed using the ACT score. This measure has previously been used in Italy, in a study conducted by the Italian Society of General Medicine (Societá Italiana di Medicina Generale) [35]. It was defined by international guidelines as: $\mathrm{ACT} \leq 14=$ not controlled, $15 \leq \mathrm{ACT} \leq 19=$ partially controlled, and ACT $\geq 20$ controlled [36]. A clinically relevant difference was defined as change from not controlled/partially controlled to controlled asthma.

Secondary outcome measures were:

1. Number of active ingredients used by patients at the point of delivery of the I-MUR provision and 3-months follow-up, as reported by patients.

2. Patients' self-reported adherence to asthma medication at the point of delivery of the I-MUR provision and 3-months follow-up, measured using two questions adapted from the Morisky Medication-Adherence Scale-MMAS (8-item) [37] embedded within the I-MUR interview.

3. Cost-effectiveness of I-MUR asthma service compared with usual care, measured in terms of cost per QALY (as a measure of disease burden, including both the quality and the quantity of life) gained, at different time points.

\section{Recruitment, inclusion criteria, randomization and blinding}

The study was conducted with the support of the Italian Pharmacists' Federation (FOFI), which supported pharmacist recruitment in each region. Eligible pharmacists, working in pharmacies with private consultation facilities and internet connection, were identified by FOFI and invited to participate in the study. Those who agreed received training in providing the I-MUR, patient recruitment and data collection by AM. Each pharmacist was instructed to recruit five adult patients with an asthma diagnosis or at least 6 months consecutive use of medicines indicating asthma.

Pharmacists within each region were randomly allocated to Group A (intervention) or B (control) by JK, using computerised random number generation in blocks of 10 . AM provided training at either T0 (Group A) or T3 (Group B), thus neither the research team nor the pharmacists were blind to delivery of the intervention. All data, comprising both primary and secondary outcome measures, were collected by the pharmacists and entered anonymously onto a web-based template, to which the researchers remained blind until an interim analysis took place at T6, which was required by the funders.

\section{Sample size, training and study-timeline}

The study aimed to involve 360 pharmacists and 1800 patients, with each pharmacist recruiting five patients. Full inclusion and exclusion criteria for both pharmacists and patients are described in the trial protocol, [33] along with details of the training provided to pharmacists and the study timeline.

Neither the pharmacist nor patient numbers anticipated were achieved, therefore it was necessary to determine the post-hoc power of the study. In the light of the number of pharmacists and patients enrolled and remaining in the study from T0 to T9, (see results), the actual power of the study was calculated maintaining the intra-cluster correlation coefficient (ICC) value of 0.02 and level of significance (two-tailed alpha) at 0.05 as originally proposed (SPSS Sample Power 3, IBM) [38].

\section{Data sources}

ACT score was obtained at all four time points, adherence and number of active ingredients on three occasions and PCIs once; more detail is available in the protocol.

Due to budget constraints primary data on cost and utility outcomes were not collected. Evidence on direct and indirect costs $[6,13]$ and health benefit (e.g. utility assessed using EuroQol 5 dimensions, EQ-5D) [39] was extrapolated from the literature and used to inform the economic evaluation (see below).

\section{Effectiveness analysis}

Effectiveness data on asthma control, number of active ingredients used, number of PCIs, patients' adherence to asthma medication were visually inspected using the quantile-quantile plot and the Kolmogorov-Smirnov test to check for normality. Data were not normally distributed therefore non-parametric techniques were used for analysis. Differences between groups A and B were compared using Mann-Whitney $U$ test for continuous variables and $X^{2}$ (Chi square) for categorical variables. Wilcoxon Sign Rank Test was used for continuous variables within the same group at different time points (T0, T3, T6 and T9) 
[40]. Variation of ACT score across T0, T3, T6 and T9 was conducted using Friedman's ANOVA, instead of one way repeated measure ANOVA as anticipated in the protocol. Correspondence analysis (CA), a multivariate analysis technique which allows graphical representation of data showing how variables are related, was used to assess the relationship between ACT scores and adherence. The primary outcome (ACT score) was analysed using two separate approaches: intention to treat (ITT) analysis, applying the last observation carried forward (LOCF), and per protocol analysis (PP). A separate analysis of primary outcome at T3, using generalised estimating equations (GEE), was conducted as recommended by Consort Statement 2010 [41] and Galbraith et al. (2010) [42] for cluster data, because GEE is a semiparametric technique used for continuous non-normal, binary and categorical responses. Moreover the Agency for Healthcare Research and Quality (AHRQ) suggests using GEE to either repeated measures at fixed intervals or for a single measure accounting for the clustering effect (multi-site study) as in our case [43]. Binary logistic analysis was used, dichotomising outcomes into not-controlled $(\mathrm{ACT} \leq 19)$ and controlled (ACT $\geq 20$ ). The GEE model was adjusted for the ACT score at baseline using age and gender. The relative risk (RR), the relative risk reduction (RRR), the absolute risk reduction (ARR) and the number needed to treat (NNT) were calculated using the PP results. An online calculator provided by the Centre for Evidence-Based Medicine of Toronto was used to confirm the results calculated manually, which also provided significance levels ( $p$ value) and $95 \%$ confidence intervals (CI) [44].

Secondary outcomes (number of active ingredients used, number of PCIs, adherence) were analysed only for patients completing the trial (PP). All analyses were conducted using SPSS software v22.

\section{Cost effectiveness analysis}

Published data from European $[6,13]$ and Italian population-based studies monitoring the annual cost [6] and quality of life [39] in terms of EQ-5D relating to ACT score were used for this analysis. Average annual cost and utility estimates per patient were extrapolated for not controlled, partially controlled, and controlled patients (see ACT score categories above) and linked to the individual patient ACT scores reported at all four time points (T0, T3, T6, and T9). Group B patients were defined as receiving usual care by keeping ACT scores measured at T3 (prior to receiving the I-MUR service) constant across time. A sensitivity analysis was conducted keeping Group B ACT scores at T3 constant across time (T6, T9). As mean ACT score values differed between the groups at baseline, changes in costs and QALY over the follow-up period considering three separate scenarios, (T3, T6 and T9) were analysed.

The primary economic analysis compared I-MUR service and usual care from the public healthcare perspective whereas the secondary analysis considered both direct and indirect costs. For the primary analysis we used Vervloet et al. (2006) [13] cost data on scheduled healthcare visits to their usual physician and specialist, unscheduled healthcare asthma related in-patient admissions, emergency visits, and emergency contacts with a physician. The secondary analysis included indirect costs on productivity losses (working days lost) and leisure time forgone (days with limited, not work-related activities) as well as direct costs on doctor visits, clinical and laboratory tests, pharmacological treatment, emergency visits, and hospital admissions, using data from Accordini et al. (2013) [6]. Costs were actualised to 2015 values using the appropriate consumer price index [45]. The cost for delivering the IMUR service to Group A at T0 was $€ 40$, calculated based on data available in the international literature [31]. The summary cost-effectiveness statistic calculated was the incremental cost-effectiveness ratio (ICER). Uncertainty and variation around the ICER mean are represented by the cost effectiveness acceptability curve (CEAC), obtained by re-sampling the data 1000 times to generate a mean cost and life year or QALY gain from each group, using a nonparametric bootstrap approach. The proportion of re-sampled datasets for which the calculated ICER lies below a given threshold is interpreted as the probability that the ICER of the intervention is below that threshold. Due to the lack of an official willingness-to-pay (WTP) threshold in Italy, we used a threshold of $€ 30,000(£ 24,017)$ per QALY, which is within the values recognized by the National Institute for Health and Care Excellence range of acceptable cost effectiveness ( $£ 20,000-30,000$ per QALY). Data analysis was performed using STATA v 13.

\section{Results}

There were 360 pharmacists randomised, of whom 283 started the study, recruiting 1263 patients at T0, with 201 pharmacists and 816 patients completing the study and having data at each time point. The median number of patients enrolled by each pharmacist was four. The overall drop-out rate from T0 to T9 was $29 \%(n=82)$ for pharmacists and 35\% $(n=447)$ for patients. The numbers in each group at T9 were 400 (49\% of the total) in group A and $416(51 \%)$ in group B (Fig. 1). Based on a $20 \%$ increase in the proportion of patients with controlled asthma (ACT score $\geq 20$ ), considering the number of individuals per cluster (4), the ICC (0.02), the calculated design effect (DE) was 1.06 , which provided an effective sample size of 758 patients, and the power attained by the study was $90 \%$.

\section{Baseline characteristics}

The numbers of pharmacists and patients from the 15 regions differed, but patient numbers recruited to Groups A and B within each region was similar. The highest 


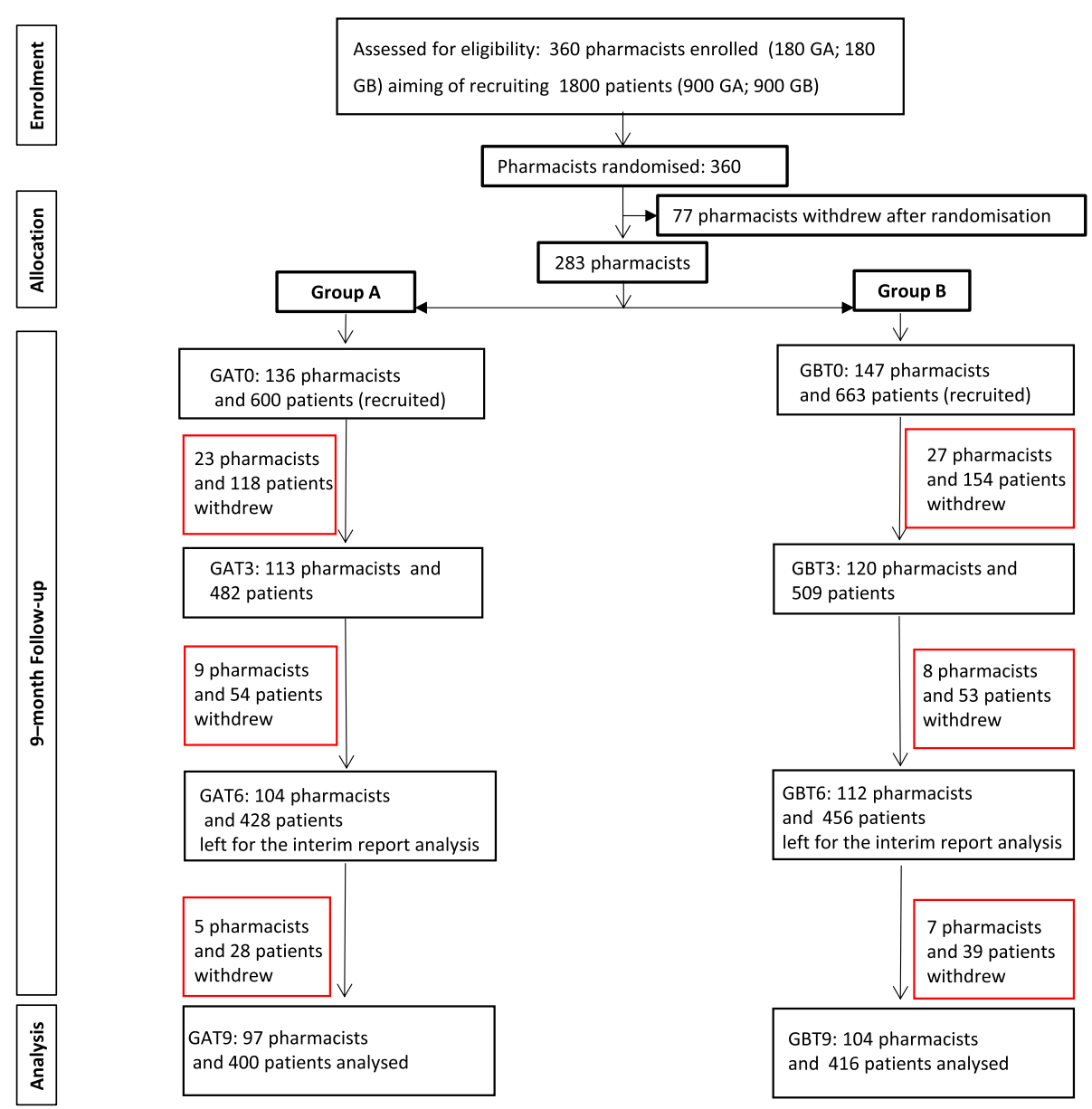

Fig. 1 CONSORT diagram describing the flow of participants though the study

number of patients was found in Sardinia and the lowest in Lazio-Campania. At T0, age range and gender showed no statistical difference between the two groups (Table 1). Overall there was a majority of female patients in both the PP $(58.82 \% ; n=480)$ and ITT analysis $(57.17 \% ; n=722)$.

\section{Process measure: pharmaceutical care issues (PCls)}

Pharmacists identified PCIs while providing the I-MUR intervention in 527 (64.58\%) patients; 277 (69.25\%) of Group A patients and 250 (60.00\%) of Group B patients. The total number of PCIs identified was 1256 , mean for the total population 1.54 per patient, and among patients with PCIs 2.40. Education required was the most frequent PCI identified in both groups, which together with monitoring issues, discrepancy between dose prescribed and dose used, potentially ineffective therapy and potential adverse reactions accounted for $64.70 \%$ of all PCIs identified.

\section{Primary outcome: asthma control}

Using a per protocol approach to analysis, the overall proportion of patients with poorly controlled asthma at baseline (T0) was 56.13\% (458/816). However median ACT score differed significantly between the two groups, being lower in Group B patients, due to a higher proportion having poor asthma control, compared to Group A patients (Table 1). At T3, asthma control in Group A patients, who had received the I-MUR intervention, showed both a statistically significant difference $(p<0.01)$ and a clinically relevant difference compared with baseline, with the median ACT score increasing from 19 (uncontrolled) to 20.50 (controlled) (Table 2). There were 41 of 205 patients $(20.00 \%)$ with ACT score $\leq 20$ who achieved control, and the proportion of patients with controlled asthma increased from 48.70 to $59.00 \%$ (Fig. 2). Group B patients, who continued to receive usual care and did not receive the I-MUR intervention until after the ACT score was obtained at $\mathrm{T} 3$, also had a statistically significant increase in ACT score $(p<0.01) ; 25 / 283$ (9.90\%) with poor or partial control achieved good control; the median ACT score increased from 18 to 19 (Table 2) and the proportion of patients with controlled asthma increased from 39.30 to $45.20 \%$ (Fig. 2). However, as the median ACT score in Group B at T3 was still below the threshold for good 
Table 1 Baseline characteristics and ACT scores

\begin{tabular}{|c|c|c|c|c|c|c|}
\hline & \multicolumn{3}{|l|}{ Recruited } & \multicolumn{3}{|l|}{ Completed } \\
\hline & Group A & Group B & $p$ & Group A & Group B & $p$-value \\
\hline Number of patients & 600 & 663 & & 400 & 416 & \\
\hline Female $\% * *$ & 58.80 & 55.70 & 0.26 & 59.80 & 57.90 & 0.60 \\
\hline \multicolumn{7}{|l|}{ Age range $n(\%)$} \\
\hline 18 to $30^{* *}$ & $55(9.17)$ & $68(10.26)$ & 0.96 & $32(8.00)$ & $43(10.34)$ & 0.69 \\
\hline 31 to $40^{* *}$ & $81(13.50)$ & $89(13.42)$ & 0.96 & $58(14.50)$ & $62(14.90)$ & 0.69 \\
\hline 41 to $50^{* *}$ & $117(19.50)$ & $122(18.40)$ & 0.96 & 79 (19.75) & $77(18.51)$ & 0.69 \\
\hline 51 to $60^{* *}$ & $114(19.00)$ & $125(18.85)$ & 0.96 & $73(18.25)$ & $80(19.23)$ & 0.69 \\
\hline 61 to $70^{* *}$ & $125(20.83)$ & $136(20.51)$ & 0.96 & $82(20.50)$ & $77(18.51)$ & 0.69 \\
\hline 71 to $80^{* *}$ & $83(13.83)$ & $88(13.27)$ & 0.96 & $59(14.75)$ & $52(12.50)$ & 0.69 \\
\hline Over $81^{* *}$ & $25(4.17)$ & $35(5.28)$ & 0.96 & $17(4.25)$ & $25(6.01)$ & 0.69 \\
\hline \multicolumn{7}{|l|}{ ACT scores } \\
\hline Median (IQR)* & $19(14.25-23)$ & $17(17-21)$ & $<0.01$ & $19(15-23)$ & $18(18-24)$ & $<0.01$ \\
\hline 5-14 not controlled $n(\%)^{* *}$ & $150(25.00)$ & $203(30.60)$ & $<0.01$ & $91(22.80)$ & $121(29.10)$ & 0.01 \\
\hline 15-19 partially controlled $n(\%)^{* *}$ & $177(29.50)$ & $223(33.60)$ & $<0.01$ & $114(28.50)$ & $132(31.60)$ & 0.01 \\
\hline 20-25 controlled $n(\%)^{* *}$ & $273(45.50)$ & 237 (35.71) & $<0.01$ & 195 (48.70) & 163 (39.30) & 0.01 \\
\hline
\end{tabular}

* Mann-Whitney $U$ test, Median (IQR), $p<0.05$

${ }^{* *}$ Chi-square test, $p<0.05$

control (ACT $\geq 20$ ), this may not be regarded as a clinically relevant change. The proportional increase in asthma control from T0 to T3 was $21 \%$ in Group A and $15 \%$ in Group B, the RR was 2, the RRR was 1.02 (95\% CI 2.21-0.28), the ARR was 0.10 (95\% CI 0.17-0.03) and the NNT was 10 (95\% CI 6-28). At T6, asthma control improved further in both groups. The increase in Group B patients, 3 months after receiving the I-MUR intervention, was both statistically significant $(p<0.01)$ and clinically significant median ACT score increasing from 19 (not controlled) to 20 (controlled) (Table 2). The proportion of Group B patients controlled increased again at T9, although the median ACT score remained the same $(p=0.05)$. Patients in Group A continued to show improvements in ACT score both at $\mathrm{T} 6(\mathrm{ACT}=21)$ and $\mathrm{T} 9$ $(\mathrm{ACT}=22)$, but only the improvement at $\mathrm{T} 9$ was statistically significant $(p<0.01)$.

Using this approach, the proportion of patients in both groups with controlled asthma continued to improve at

Table 2 Median ACT scores plus inter quartile range (IQR) for Group A and Group B using both PP and ITT analyses

\begin{tabular}{llllll}
\hline & & T0 & T3 & T6 & T9 \\
\hline PP & GA & $19(15-23)$ & $20.5(17-23)$ & $21(17-24)$ & $22(18-24)$ \\
& GB & $18(14-22)$ & $19(15-22)$ & $20(16-22.75)$ & $20(16-23)$ \\
ITT & GA & $19(14.25-23)$ & $20(16-23)$ & $21(16-23)$ & $21(17-24)$ \\
& GB & $17(17-21)$ & $18(14-21)$ & $19(15-22)$ & $20(15-22)$ \\
\hline
\end{tabular}

Intervention was delivered immediately after ACT at T0 in Group A and immediately after ACT at T3 in Group B, thus T6 scores are 6- and 3-months post-intervention for groups $A$ and $B$ respectively; T9 scores are 9 - and 6-months post-intervention for groups $A$ and $B$ each time point, but the median ACT score only reached a value indicating controlled asthma in both groups following the I-MUR intervention. The increase was greater in group B $(29.00 \%)$ compared to group A $(21.00 \%)(p<0.01)$. From T0 to T9 the percentage increase in controlled patients was $40.20 \%$ and $45.00 \%$ respectively in Groups A and B. Thus asthma control was not only sustained but continued to increase throughout the study (Fig. 2).

Including all patients in an ITT analysis showed an even greater difference between the groups at baseline in the proportion with asthma control (ACT score $\geq 20$ ) and that the median ACT score did not reach $\geq 20$ for patients in Group B until T9, whereas patients in Group A achieved this at T3. However, as with the per protocol analysis, ACT score showed an overall increase in both groups at each time point ( $p<0.01$; Friedman's ANOVA). The result of the GEE analysis applied to PP showed that the I-MUR intervention resulted in improved asthma control (ACT scores at T0 versus ACT scores at T3): patients receiving the intervention were 1.8 times more likely to improve from not controlled $(\mathrm{ACT} \leq 19)$ to controlled $(\mathrm{ACT} \geq 20)$ than control patients (odds ratio (OR) 1.76, 95\% CI 1.33-2.33); the ICC was 0.07 (95\% CI $0.008-$ 0.168). A similar result was obtained also when a more stringent and conservative approach was used during the ITT analysis (OR 1.70, 95\% CI 1.36-2.13).

\section{Secondary outcomes} Number of active ingredients

Data on asthma-related medicines regularly used by patients were gathered during the I-MUR intervention, at 

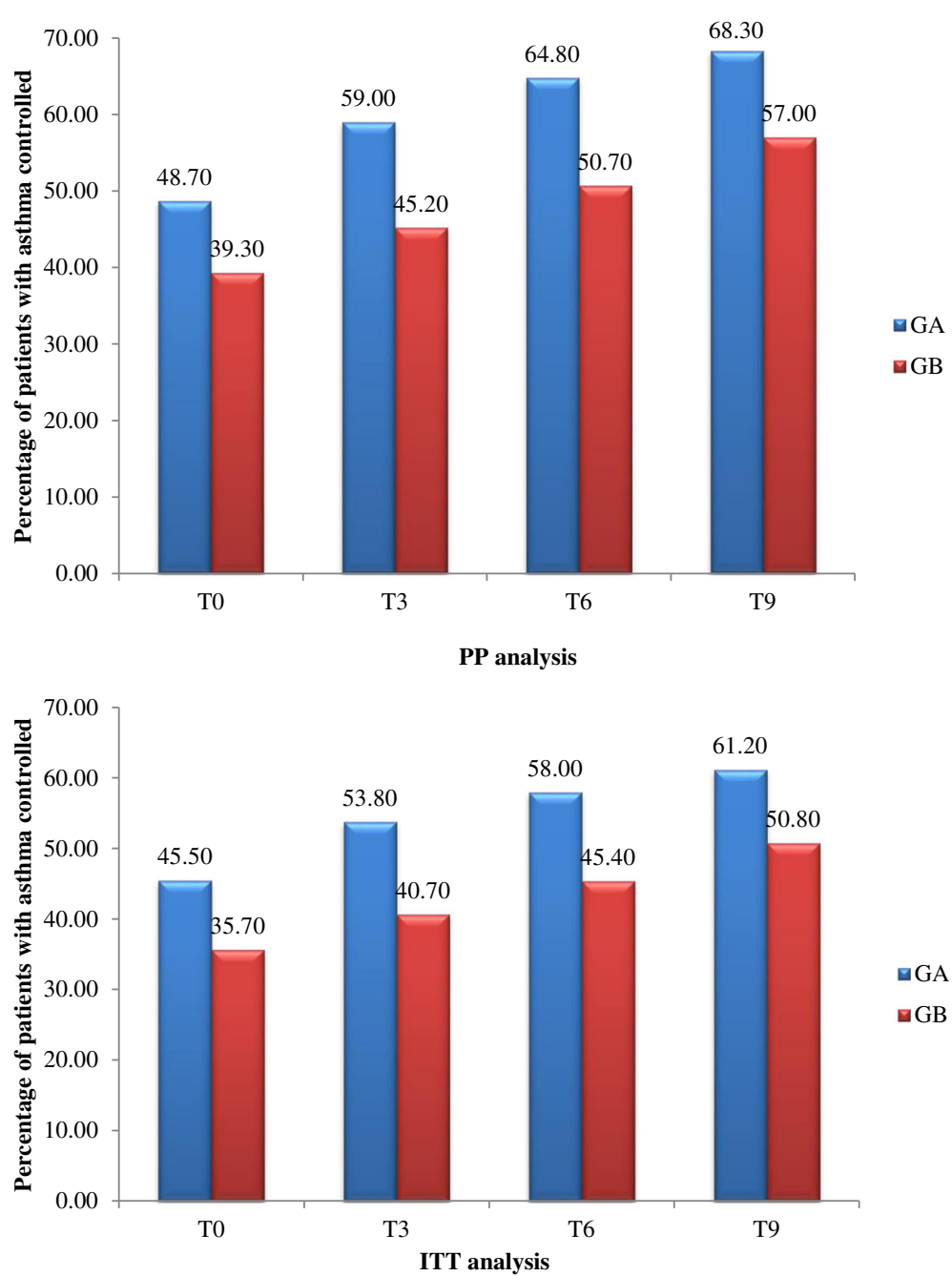

Fig. 2 Percentage of patients in group A and B with controlled asthma (ACT score $\geq 20$ ) at four time points shown as both PP and ITT

T0 for group A and at T3 for group B. There were no significant statistical differences between the two groups. The total number of active ingredients recorded by pharmacists as being used in 812 patients was 4310 , median 5 per patient, range $0->20$. After the I-MUR intervention, the total number of active ingredients was $3970(n=759)$, median 4 , range $1-20$, a reduction of $n=340$ or $7.90 \%(p<0.01)$. The reduction was maintained in both groups 6 months after the intervention (Table 3).

Table 3 Changes in the number of active ingredients used by patients before and after the I-MUR intervention

\begin{tabular}{llll}
\hline & Before I-MUR & 3 months after I-MUR & 6 months after I-MUR \\
\hline GA & $5(3-7)$ & $4(3-7)$ & $4(3-7)$ \\
GB & $5(3-7)$ & $4(2-7)$ & $4(3-7)$ \\
\hline
\end{tabular}

Values are presented as median (IQR)

\section{Adherence}

Self-reported adherence was recorded in 802 patients on three occasions during the study, using questions adapted from Morisky scale. Significant $(p<0.01)$ improvements were found in both groups at both 3 and 6 months post intervention, compared to before the I-MUR. The greatest improvement in adherence occurred at 3 months post-intervention, with the improvement sustained at 6 months (Fig. 3).

Correspondence analysis demonstrated that the patients who achieved asthma control (ACT 20-25) were those who did not miss or change their medication (Fig. 4).

\section{Cost effectiveness analysis}

At 3-months follow-up, only the difference in costs (from the public healthcare and society perspectives) were significant at the 0.01 level (Table 4). The bootstrap 


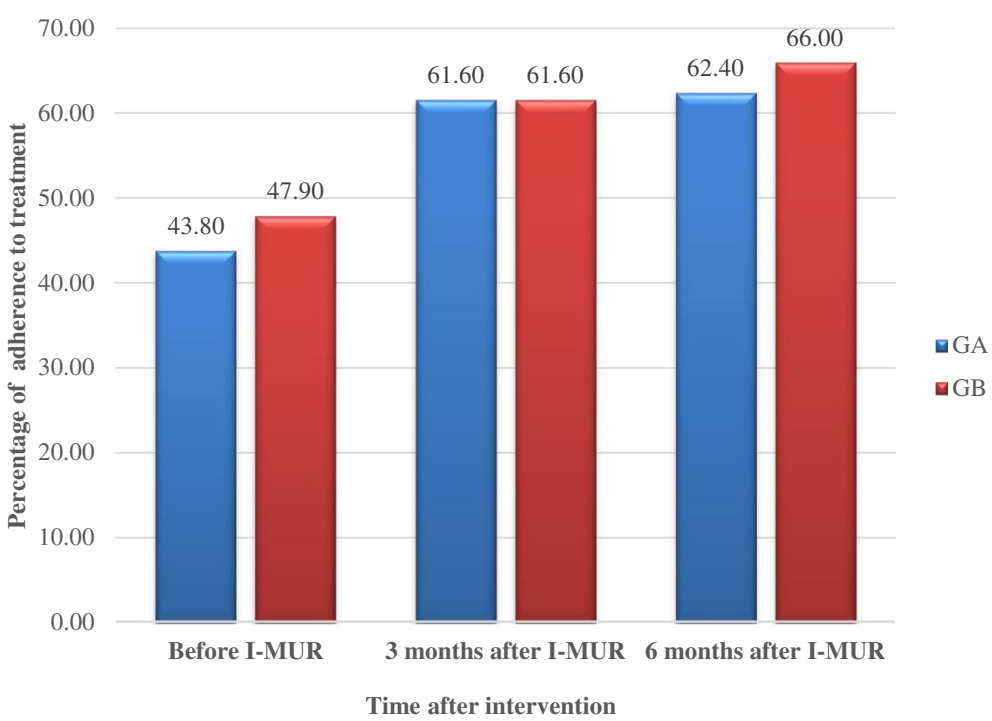

Fig. 3 Patients' self-reported adherence to medications before and after I-MUR

method was therefore employed to evaluate uncertainty surrounding cost-effectiveness estimates.

In the economic analysis we have used mean rather than median values, as this is more informative for planners of healthcare services. As the patient cost data were skewed, the confidence limits around the means were generated using non-parametric bootstrapping techniques. The incremental cost-effectiveness plane (CEP) showed $60.50 \%$ of the plots falling into the south-east quadrant, indicating that I-MUR service dominated the usual care option (less costly and more effective). Considering a WTP threshold of $€ 30,000$ per additional QALY gained, the probability that I-MUR was more cost-effective is $71.50 \%$. For the secondary analysis, the incremental CEP showed about half of the plots falling into the south-east quadrant. The probability that I-MUR care was more cost-effective was $51.50 \%$ (Figs. 5 and 6). At 6 months, both the difference in costs and utility were significant at 0.01 level. The majority of plots in the cost-effectiveness scatter fell in the southeast quadrant (between 82.70 and 69.60\%; public healthcare and society perspectives, respectively) and indicated that usual care was dominated by I-MUR service. The probability that I-MUR care was more cost-effective varied between 93.00 and $89.50 \%$, respectively (Figs. 5 and 6).

At 9 months, both the difference in costs and utility were significant at 0.01 level. The large majority of plots

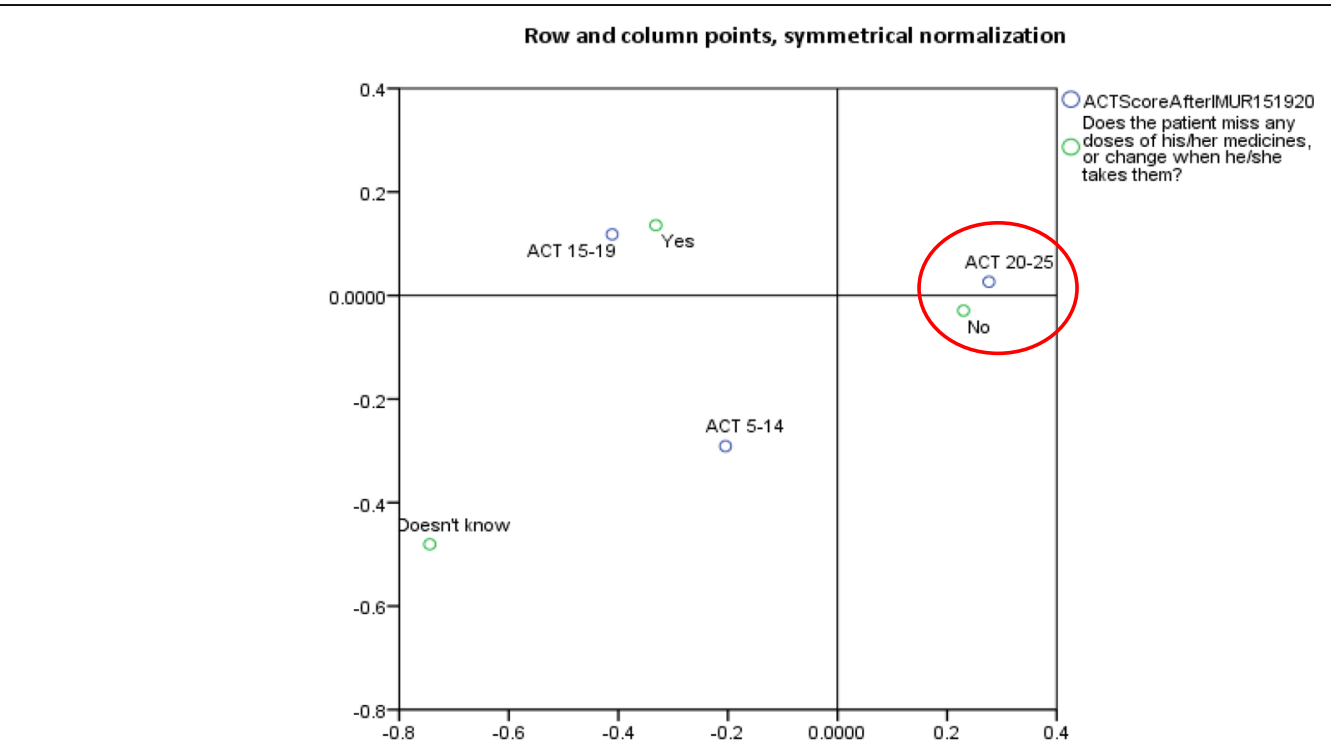

Fig. 4 Relationship between asthma control and adherence to treatment after I-MUR 
Table 4 Costs and QALY estimates for the three scenarios considered in the cost-utility analysis

\begin{tabular}{|c|c|c|c|c|c|}
\hline & \multicolumn{2}{|c|}{$\begin{array}{l}\text { Intervention } \\
(N=600)\end{array}$} & \multicolumn{2}{|l|}{$\begin{array}{l}\text { Control } \\
(N=663)\end{array}$} & \multirow{2}{*}{$\begin{array}{l}p \text {-value } \\
P \\
\end{array}$} \\
\hline & Mean & Sd & Mean & Sd & \\
\hline \multicolumn{6}{|l|}{ Scenario 1: 3 months } \\
\hline Difference in yearly patient costs between T3 and T0 (public healthcare perspective, [13] Euros 2015) & -122.63 & 747.03 & -113.29 & 828.04 & 0.01 \\
\hline Difference in yearly patient costs between T3 and T0 (societal perspective, [6] Euros 2015) & -95.17 & 660.94 & -95.82 & 741.32 & 0.01 \\
\hline Difference ${ }^{1}$ in QALYs between T3 and T0 [39] & 0.02 & 0.10 & 0.01 & 0.10 & 0.86 \\
\hline \multicolumn{6}{|l|}{ Scenario 2: 6 months } \\
\hline Difference in yearly patient costs between T6 and T0 (public healthcare, [13] Euros 2015) & -154.84 & 846.47 & -113.29 & 828.04 & 0.01 \\
\hline Difference in yearly patient costs between T6 and T0 (societal perspective, [6] Euros 2015) & -115.93 & 741.45 & -95.82 & 741.32 & 0.01 \\
\hline Difference in QALY s between T6 and T0 [39] & 0.03 & 0.12 & 0.01 & 0.10 & 0.01 \\
\hline \multicolumn{6}{|l|}{ Scenario 3: 9 months } \\
\hline Difference in yearly patient costs between T9 and T0 (public healthcare perspective, [13] Euros 2015) & -207.04 & 828.88 & -113.29 & 828.04 & 0.01 \\
\hline Difference in yearly patient costs between T9 and T0 (societal perspective [6] Euros 2015) & -158.56 & 729.97 & -96.12 & 741.37 & 0.01 \\
\hline Difference in QALYs between T9 and T0 [39] & 0.04 & 0.11 & 0.01 & 0.10 & 0.01 \\
\hline
\end{tabular}

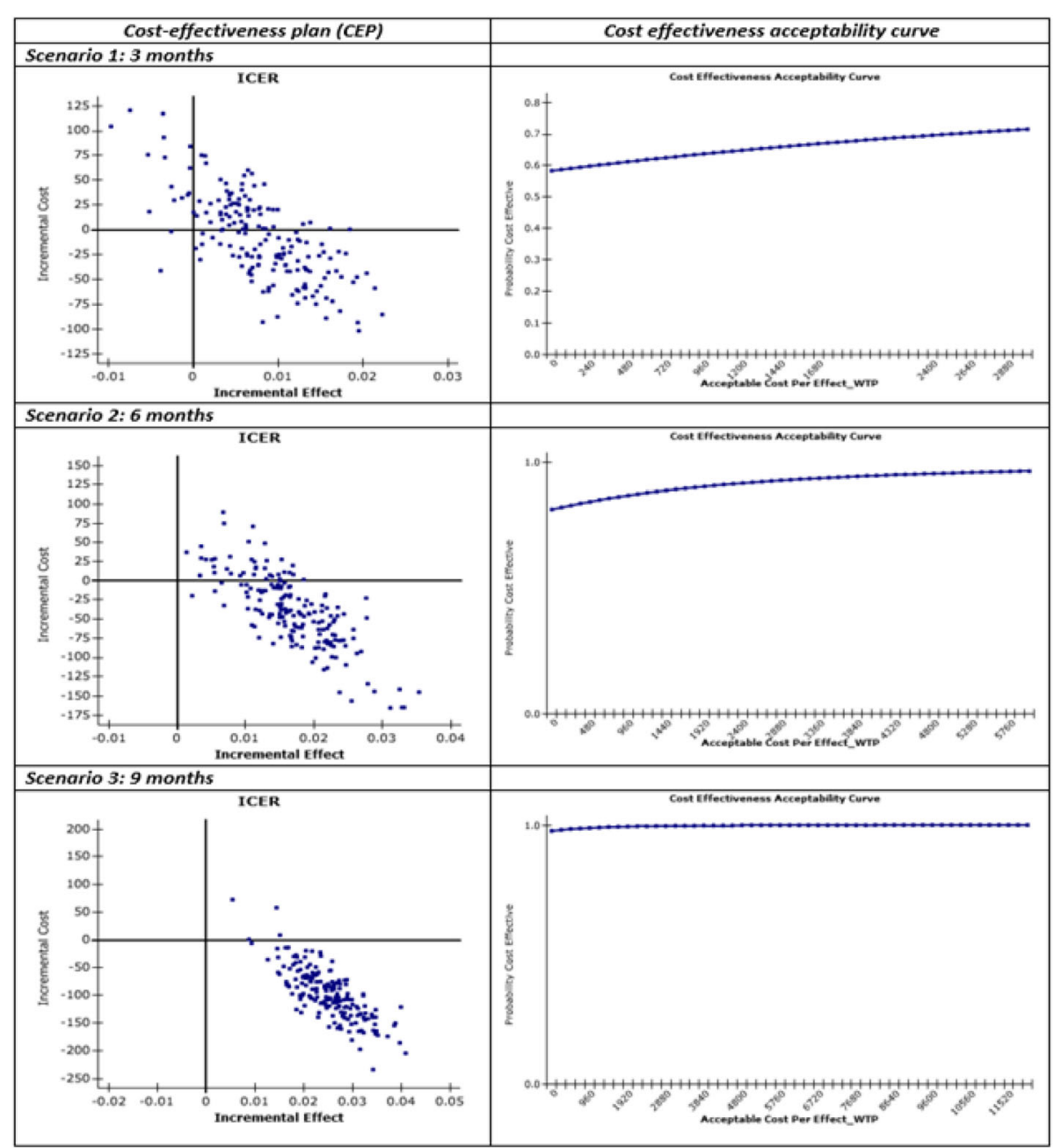

Fig. 5 Cost Utility analysis (Italian public healthcare perspective, Euros 2015) 


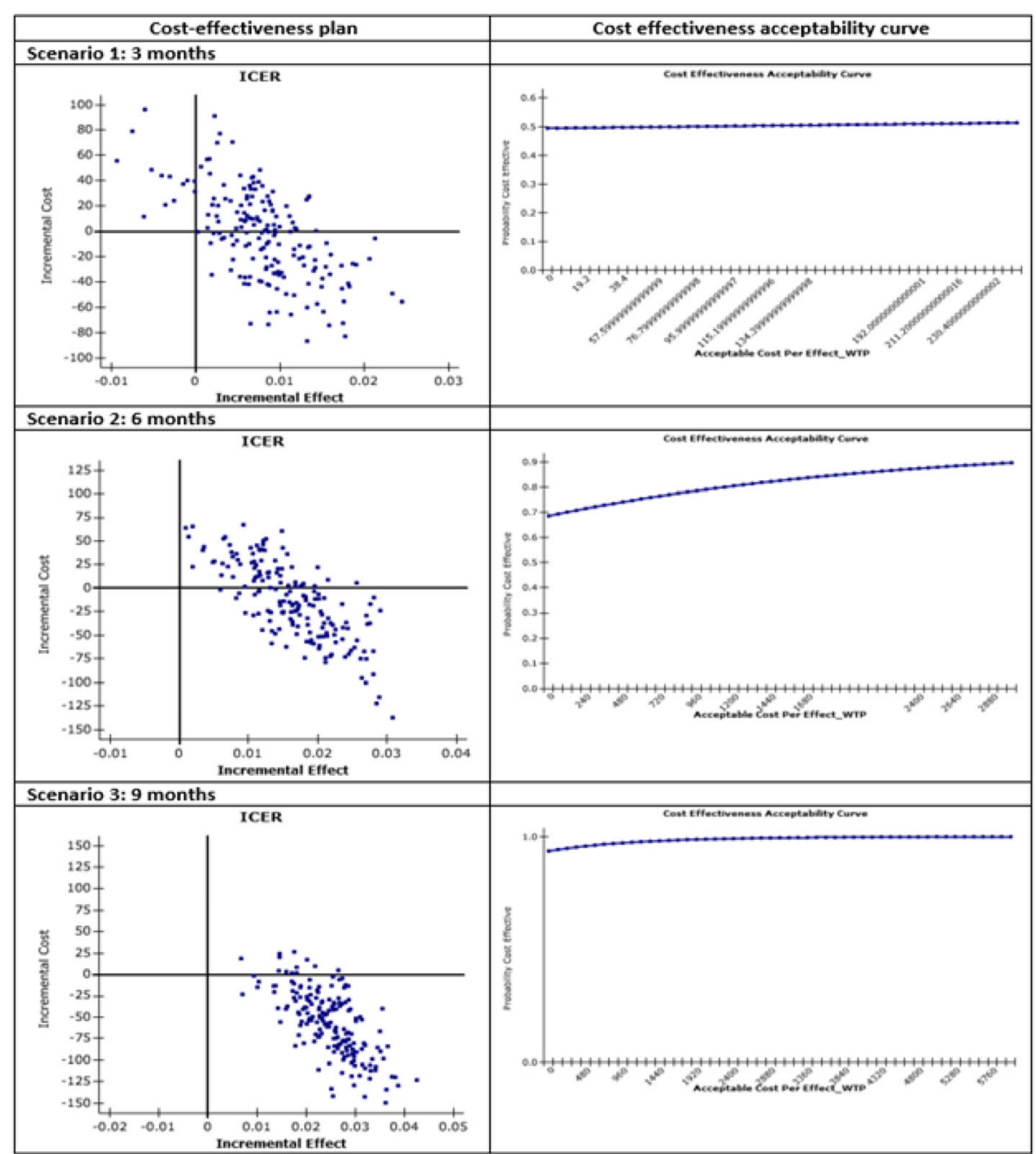

Fig. 6 Cost utitlity analysis (society perspective, Euros 2015)

in the cost-effectiveness scatter fell in the south-east quadrant (between 98.10 and $93.60 \%$; public healthcare and society perspectives, respectively) and indicated that usual care was dominated by I-MUR service. The probability of I-MUR being cost-effective was $100 \%$ (Figs. 5 and 6). Overall the probability of I-MUR care being cost-effective doubled across time (from $51.50-71.50 \%$ at 3 months up to $100 \%$ at 9 months).

\section{Discussion}

\section{Main findings}

The effectiveness analysis showed evidence for the superiority of I-MUR service compared with usual care for patients with asthma in terms of the primary outcome of ACT score and all of the secondary outcomes. There was a statistically significant improvement in the proportion of patients with controlled asthma in both groups over the 9 months of follow-up and the median ACT score moved from partial to good control after the I-MUR intervention, at T3 in Group A and T6 in Group B. The NNT was 10, thus indicating a potentially useful intervention. Relatively few studies of pharmacist interventions report NNTs, however of those which do, values are similar to that found here $[46,47]$.

The I-MUR intervention was shown to reduce the number of active ingredients being used and to improve patients' adherence to their medications. In addition, a clear link was demonstrated between adherence and ACT score, suggesting that this may be one mechanism whereby asthma control was improved. Adherence increased overall by $35.4 \% 3$ months post-intervention and by $40.0 \%$ at 6 months. The types of pharmaceutical care issues identified by the pharmacists during the I-MUR were primarily related to the need for education, monitoring and potentially ineffective therapy. Hence the provision of advice on use of medicines and how to optimise their effectiveness, together with frequent monitoring is likely to be the mechanism whereby adherence and thus asthma 
control were improved. The continuous improvement in both asthma control and adherence in both groups suggests that regular contact with the pharmacist and completion of the ACT score was in itself an important factor.

Cost-effectiveness analysis supported the hypothesis that I-MUR is more cost effective than usual care and the probability of I-MUR being cost-effective doubled across time (from $51.50 \%$ at 3 months to $100 \%$ at 9 months). Moreover it was demonstrated that the key factor influencing asthma control was adherence, which pharmacists are well placed to influence, and the resultant improvements in both were sustained over at least 6 months (9 months in Group A).

\section{Comparison with other studies}

Similar results have been found in RCTs of community pharmacists' intervention in asthma in other countries, across Europe [27, 28], Australia [26] and North America [22], with the largest studies to date being conducted in Australia, Belgium and Spain [26-28]. One Australian study [48] showed both improved adherence and improved asthma control which was sustained for 12 months. The Belgian study [27] assessed adherence through prescription refill rates rather than patient completed questions, but also assessed patient knowledge about asthma medication, but showed no change in asthma control overall, only in a subgroup of patients with uncontrolled asthma. While the Spanish study [28] showed evidence of benefit, the work was funded by a pharmaceutical company and one of the patients' inclusion criteria was the use of Budesonide/Formoterol, a medication produced by the same company.

Other studies have also shown potential benefits of pharmacist intervention in asthma, although many were small and some were uncontrolled [29]. The English MUR service, on which this intervention was based, has relatively little evidence to support its effectiveness [49] or cost effectiveness [50]. However one small, uncontrolled study has shown that the proportion of patients with controlled asthma rose from 41 to $55 \%$ after receiving an MUR [51]. A systematic review of fee-for-service pharmacist-led medication reviews in general showed that this type of service improves adherence and clinical markers in a range of conditions [52]. It is essential that future studies should incorporate both clinical outcomes and economic evaluations, to enable governments and other commissioners to assess the potential benefits of such services.

\section{Strengths and limitations}

This is the first study in Italy to evaluate a community pharmacist-based intervention, and, to the best of our knowledge, it appears to be the largest RCT of a community pharmacy intervention in asthma conducted in any country in terms of the numbers of pharmacists and patients. Further strengths are that it adopted the ACT score as a measure of asthma control, as recommended by GINA, which allowed measurement of both effectiveness and cost-effectiveness (by translating ATC scores into QALYs) of the novel I-MUR intervention. The large number of pharmacists involved throughout the country supports the generalisability of the results at national level.

Standard practice in community pharmacy in Italy involves little clinical input, being mainly a supply function, therefore the patients were unused to a pharmacist taking an interest in their clinical status and providing information about optimising medicines use, which may have contributed to the effectiveness of the intervention. The pharmacists providing the I-MUR were responsible for both selecting patients and entering the data collected onto the electronic template, possible sources of bias. The population overall included more females than males $(p<0.01)$ and, more importantly, the proportion of patients not controlled differed between groups $\mathrm{A}$ and $\mathrm{B}$, with median ACT scores at baseline of 19 and 18. The assessment of adherence did not use a validated tool, but instead used only two patient-reported questions, in the interests of brevity, which were similar to the questions used in the English MUR template. Also the initial follow-up period before the usual care group received the intervention was only 3 months. This meant that we had to assume no change in ACT control from T3 onwards for the economic analysis. In addition it was necessary to use secondary data rather than primary data collection on cost and utility, which were derived from different sources and were old estimates [6, 13], actualised at 2015 [45].

\section{Conclusion}

This study demonstrates that the I-MUR service, which is the first cognitive pharmaceutical service to be delivered in Italy, was both effective and cost-effective. The Italian Government/Ministry of Health have since promoted a change of community pharmacy practice, with the I-MUR being the first nationally funded cognitive pharmaceutical service in Italy [53]. The work has therefore supported a significant cultural shift in Italian community pharmacy practice, promoting the change from a mainly logistic to a more patient-centred and clinically-oriented role of the community pharmacist in delivery of health care. Consideration is being given to extending the service to other respiratory conditions, but the successful service model applied here could enable community pharmacists in Italy to support the care of patients with a wide range of long-term conditions in the future. Moreover, it adds to the evidence base world-wide demonstrating the potential effectiveness and cost-effectiveness of community pharmacy medication reviews. 


\section{Abbreviations}

95\% Cl: 95\% Confidence interval; ACT: Asthma control test; AHRQ: Agency for healthcare research and quality; ARR: Absolute risk reduction; CA: Correspondence analysis; CEAC: Cost-effectiveness acceptability curve; CEP: Cost-effectiveness plane; DALYs: Disability-adjusted life years; DE: Design effect; ESS: Effective sample size; EuroQol-5D: Survey instrument for describing health-related quality of life; FOFI: Italian Pharmacists' Federation/ Federazione Ordini Farmacisti Italiani; GEE: General estimating equations; GINA: Global initiative for asthma; IBM: International business machine; ICC: Intra-cluster correlation coefficient; ICER: Incremental cost-effectiveness ratio; I-MUR: Italian medicines use review; ITT: Intention to treat; LOCF: Last observation carried forward; m: Number of units (patients) per cluster; MMAS8: Eight item morisky medication adherence scale; MUR: Medicines use review; NNT: Number needed to treat; OR: Odds ratio; PCls: Pharmaceutical care issues; PI: Principal investigator; PP: Per protocol; QALY: Quality-adjusted life-year; RCT: Randomised controlled trial; RR: Relative risk; RRR: Relative risk reduction; SIMG: Italian Society of General Medicine/Societá Italiana di Medicina Generale; SPSS: Statistical package for the social sciences; STATA: Statistics data analysis; T0: Time zero; T3: Time at three months; T6: Time at six months; T9: Time at nine months; WTP: Willingness-to-pay

\section{Acknowledgements}

The authors are grateful to the Italian pharmacists, general practitioners and consultants, patients and all other people who have been involved in this study. The study protocol can be accessed at http://dx.doi.org/10.1186/ s12913-015-0791-6

\section{Funding}

This trial was funded by the Italian Pharmacists' Federation (FOFI) and the trial protocol is registered with ISRCTN (ISRCTN72438848). The funder played no role in the study design and the collection, analysis, and interpretation of data and the writing of the article and the decision to submit it for publication. Medway School of Pharmacy funded MT for conducting the cost-effectiveness analysis. The research conducted was independent of any involvement from the sponsors of the study. Study sponsors were not involved in study design, data interpretation, writing of the manuscript, or the decision to submit the article for publication.

\section{Availability of data and materials}

Data supporting the conclusions of this article can be found in the University of Kent Academic Repository (KAR).

\section{Authors' contributions}

Andrea Manfrin (AM) was the PI who conceived and designed the study with Janet Krska (JK) and Trudy Thomas (TT). AM developed the I-MUR instrument, monitored the recruitment process and carried out data collection using the web platform, performed data coding and effectiveness analysis. JK supervised the work conducted by AM. Michela Tinelli (MT) designed and conducted the health economics analysis, and all authors interpreted the results. AM, JK and MT drafted the manuscript. TT reviewed the manuscript. All authors revised the manuscript for intellectual content, read and approved the final manuscript. The researchers had access to all data. AM acts as the guarantor for this study.

\section{Competing interests}

The authors declare that they have no competing interests.

\section{Consent for publication}

Written consent for publishing the results was obtained from patients and pharmacists before their enrolment in the study.

\section{Ethics approval and consent to participate}

This study was conducted according to the principles expressed in the Declaration of Helsinki, it was approved by the University of Kent Faculty of Sciences Research Ethics Advisory Group for Human Participants on February $18^{\text {th }} 2014$ (reference No 0281314) and subsequent approved by the Brescia Ethics committee in Italy on June $3^{\text {rd }} 2014$ (reference No 1710-Studio RE I-MUR) which acted also as the coordinating centre in Italy. Written consent for participation in the study was obtained from patients and pharmacists before their enrolment in the study.

\section{Publisher's Note}

Springer Nature remains neutral with regard to jurisdictional claims in published maps and institutional affiliations.

\section{Author details}

${ }^{1}$ Medway School of Pharmacy, Universities of Greenwich and Kent at Medway, Anson Building, Central Avenue, Chatham Maritime, Chatham, Kent ME4 4TB, UK. '2LSE Health and Social Care, The London School of Economics and Political Science, Houghton Street, London WC2A 2AE, UK.

Received: 14 April 2016 Accepted: 12 April 2017

Published online: 24 April 2017

\section{References}

1. De Marco R, Cappa V, Accordini S, Rava M, Antonicelli L, Bortolami O, et al. Trends in the prevalence of asthma and allergic rhinitis in Italy between 1991 and 2010. Eur Respir J. 2012;39:883-92.

2. Masoli M, Fabian D, Holt S, Beasley R. Global Burden of Asthma. The global burden of asthma: executive summary of the GINA Dissemination Committee Report. Allergy. 2004;59(5):469-78. http://ginasthma.org/local/ uploads/files/GINABurdenSummary_1.pdf (2003). Accessed 20 Oct 2015.

3. European Respiratory Society. European Lung White book. The economic burden of lung disease. http://www.erswhitebook.org/chapters/theeconomic-burden-of-lung-disease/ (2016). Accessed 10 Sept 2015.

4. Gupta R, Sheikh A, Strachan DP, Handerson HR. Burden of allergic disease in the UK: secondary analyses of national databases. Clin Exp Allergy. 2004;34:520-6.

5. Anandan C, Gupta R, Simpson CR, Fishbacher C, Sheikh A. Epidemiology and disease burden from allergic disease in Scotland: analyses of national databases. J R Soc Med. 2009;102:431-42.

6. Accordini S, Corsico AG, Braggion M, Gerbase MW, Gislason D, Gulsvik A, et al. The cost of persistent asthma in Europe: an international populationbased study in adults. Int Arch Allergy Immunol. 2013;160:93-101.

7. Accordini SM, Bugiani Arossa W, Gerzeli S, Marinoni A, Olivieri M, et al. Poor control increases the economic cost of asthma. A multicentre populationbased study. Int Arch Allergy Immunol. 2006;141:189-98.

8. D’Amato G, Liccardi G, Beghé B, Carrozzi L, Mapp C, Braido F, et al. Libra. Global Initiative for Asthma (GINA). Italian guideline updated. http:// docslide.it/documents/progetto-mondiale-asma-linee-guida-italianeaggiornamento-2007-ferrara-8-10-marzo-2007-g-in-a-lobal-itiative-for-sthma. html. Italian (2006). Accessed 15 Dec 2015.

9. Fitzgerald MJ, Reddel H, Boulet LP, Hurd S. Pocket guide for asthma management and prevention (Global Initiative for Asthma). A pocket guide for Physicians and Nurses, Updated 2015. http://ginasthma.org/wp-content/ uploads/2016/01/GINA_Pocket_2015.pdf. Accessed 13 Dec 2015.

10. Scottish Intercollegiate Guidelines Network (SIGN 141). British guideline on management of asthma. A national clinical guideline. Healthcare Improvement Scotland. www.sign.ac.uk/pdf/SIGN141.pd (2014). Accessed 8 Jan 2016.

11. National Institute for Health Care Excellence (NICE). Asthma NICE quality standard [QS25] February 2013. https://www.nice.org.uk/guidance/qs25. Accessed 12 Jan 2016

12. European Medicines Agency (EMEA). Clinical efficacy and safety: Respiratory system? Guideline on the clinical investigation of medicinal products for the treatment of asthma. Oct 2015 CHMP/EWP/2922/01 Rev. 01;3-4. http://www. ema.europa.eu/ema/index.jsp?curl=pages/regulation/general/general_ content_000426.jsp\&mid=WC0b01ac0580034cf6. Accessed 15 Jan 2016.

13. Vervloet $D$, Williams $A E$, Lloyd A, Clark TJH. Costs of managing asthma as defined by a derived asthma control test score in seven European countries. Eur Respir Rev. 2006;15(98):17-23.

14. Braido F, Baiardini I, Balestracci S, Fassio O, Ravera S, Belotti M, et al. The relationship between asthma control and quality-of-life impairment due to chronic cough: a real-life study. Ann Allergy Asthma Immunol. 2008;101(4):370-4.

15. Demoly P, Annunziata K, Gubba E, Adamek L. Repeated cross-sectional survey of patient-reported asthma control in Europe in the past 5 years. Eur Respir Rev. 2012;21(123):66-74.

16. Allemann S, van Mill JW, Boterman L, Berger K, Griese N, Hersberger KE. Pharmaceutical care: the PCNE definition 2013. Int J Clin Pharmacol. 2014;36(3):544-55.

17. Barbanel D, Eldridge S, Griffiths C. Can a self-management programme delivered by a community pharmacist improve asthma control? A randomised trial. Thorax. 2003;58(10):851-4. 
18. Bunting BA, Cranor CW. The Asheville project: long-term clinical, humanistic, and economic outcomes of a community-based medication therapy management program for asthma. J Am Pharm Assoc. 2006;46(2):133-47.

19. Cordina M, McElnay JC, Hughes CM. Assessment of a community pharmacy-based program for patients with asthma. Pharmacotherapy. 2001;21(10):1196-203.

20. Emmerton L, Shaw J, Kheir N. Asthma management by New Zealand pharmacists: a pharmaceutical care demonstration project. J Clin Pharm Ther. 2003;28(5):395-402.

21. Mangiapane S, Schulz M, Mühlig S, IHle P, Schubert I, Waldman HC. Community pharmacy-based pharmaceutical care for asthma patients. Ann Pharmacother. 2005;39(11):1817-22.

22. McLean W, Gillis J, Waller R. The BC community pharmacy asthma study: a study of clinical, economic and holistic outcomes influenced by an asthma care protocol provided by specially trained community pharmacists in British Columbia. Can Respir J. 2003;10:195-202.

23. Narhi U, Airaksinen M, Tanskanen P, Erlund $H$. Therapeutic outcomes monitoring by community pharmacists for improving clinical outcomes in asthma. J Clin Pharm Ther. 2000;25:177-83.

24. Saini B, Krass I, Armour C. Development, implementation, and evaluation of a community pharmacy-based asthma care model. Ann Pharmacother. 2004;38:1954-60.

25. Schulz M, Verheyen F, Mühlig S, Müller JM, Mühlbauer K, Knop-Schneickert $E$, et al. Pharmaceutical care services for asthma patients: a controlled intervention study. J Clin Pharmacol. 2001;41:668-76.

26. Armour C, Bosnic-Anticevich S, Brillant M, Burton D, Emmerton L, Krass I, et al. Pharmacy asthma care program (PACP) improves outcomes for patients in the community. Thorax. 2007;62:496-502.

27. Mehuys E, Van Bortel L, De Bolle L, Van Tongelen I, Annemans L, Remon JP, et al. Effectiveness of pharmacist intervention for asthma control improvement. Eur Respir J. 2008;31(4):790-9.

28. García-Cárdenas V, Sabater-Hernández D, Kenny P, Martinez-Martinez F, Faus MJ, Benrimoj SI. Effect of a pharmacist intervention on asthma control. A cluster randomised trial. Respir Med. 2013;107(9):1346-55.

29. Garcia-Cardenas V, Armour C, Benromoj SI, Martinez-Martinez F, Rotta I, Fernandez-Llimos F. Pharmacists' interventions on clinical asthma oucomes: a systematic review. Eur Respir J. 2016;47:1043-6.

30. Pharmaceutical Service Negotiating Committee (PSNC). http://psnc.org.uk/ services-commissioning/advanced-services/murs/. Accessed 20 Jan 2016.

31. Houle SKD, Grindord AK, Chatterly T, Tsuyuki RT. Paying pharmacists for patient care. A systematic review of remunerated pharmacy clinical services. Can Pharm J. 2014;147(4):209-32.

32. Gums T, Carter B, Foster E. Cluster randomised controlled trials for pharmacy practice research. Int J Clin Pham. 2016;38(3):607-14.

33. Manfrin A, Thomas T, Krska J. Randomised evaluation of the Italian medicines use review provided by community pharmacists using asthma as a model (RE I-MUR). BMC Health Serv Res. 2015;15:171. http://dx.doi.org/10. 1186/s12913-015-0791-6. Accessed 15 Dec 2015

34. Krska J, Cromarty JA, Arris F, Jamieson D, Hansford D. Providing pharmaceutical care using a systematic approach. Pharm J. 2000;265:656-60

35. Bettoncelli G, Magnoni MG, Fassari C, Tosatto R, Di Blasi P, De Marco R et al. (POINT OUT ASTHMA CONTROL ITALIAN SURVEY) II controllo dell'asma in Italia misurato con Act A(Asthma Control Test) STUDIO P.A.C.I.S. (Point out Asthma Control Italian Survey) II controllo dell'asma in Italia misurato con ACT (Asthma Control Test). https://www.simg.it/documenti/rivista/2006/06_ 2006/3.pdf. Accessed 10 March 2014.

36. Thomas M, Kay S, Williams A, Pike J, Williams A, Carranza JR, et al. The asthma control test (ACT) as a predictor of GINA guideline-defined asthma control: analysis of a multinational cross-sectional survey. Prim Care Respir J. 2009;18:41-9.

37. Morisky DE, Alfonso A, Krousel-Wood M, Ward HJ. Predictive validity of a medication adherence measure in outpatient setting. J Clin Hypertens. 2008;10(5):348-54

38. Killip S, Mahfoud Z, Pearce K. What is an intracluster correlation coefficent? crucial concepts for primary care researchers. Ann Fam Med. 2004;2(3):204-8.

39. Terzano C, Cremonesi G, Gibrino G, Ingrassia E, Marsico S, Nicolini G, et al. 1-Year prospective real life monitoring of asthma control and quality of life in Italy. Respir Res. 2012;13:112. http://respiratory-research.com/content/13/ 1/112. Accessed12 Jan2016.

40. McCrum-Gardner E. Which is the correct statistical test to use? Br J Oral Maxillofac Surg. 2008;46(1):38-41.
41. Campbell MK, Piaggio G, Elbourne DR, Altman DG. Consort 2010 statements: extension to cluster randomised trials. Research methods and reporting. BMJ. 2012;345:e5661.

42. Galbraith S, Daniel JA, Vissel B. A study of cluster data and approaches to its analysis. J Neurosci. 2010;30(32):10601-8.

43. Velentgas P, Dreyer NA, Nourjah P, Smith SR, Torchia MM, eds. Developing a Protocol for Observational Comparative Effectiveness Research: A User's Guide. AHRQ Publication No. 12(13)-EHC099. Rockville, MD: Agency for Healthcare Research and Quality. 2013. https://www.ncbi.nlm.nih.gov/books/ NBK126190/pdf/Bookshelf_NBK126190.pdf. Accessed 12 Dec 2015.

44. The Canadian Institute of Health research (CIHR). Centre for Evidence-Based Medicine Toronto. KT Clearinghouse. http://ebm-tools.knowledgetranslation. net/calculator/randomized/. Accessed 10 Jan 2016.

45. European Central Bank. Statistical Data Warehouse. Eyrosystem. http://sdw. ecb.europa.eu/. Accessed 20 Jan 2016.

46. Meid AD, Lampert A, Burnett A, Seidling HM, Haefeli WE. The impact of pharmaceutical care interventions for medication underuse in older people: a systematic review and meta-analysis. Br J Clin Pharmacol. 2015;80:768-76.

47. Wu JYF, Leung WYS, Chang S, Lee B, Zee B, Tong P, Chan J. Effectiveness of telephone counselling by a pharmacist in reducing mortality in patients receiving polypharmacy: randomised controlled trial. BMJ. 2006;333:522.

48. Armour CL, Reddel HK, LeMay KS, Saini B, Smith LD, Bosnic-Anticevich SZ, et al. Feasibility and effectiveness of an evidence-based asthma service in Australian community pharmacies: a pragmatic cluster randomized trial. J Asthma. 2013;50(3):302-9.

49. Wright D. 2016. A rapid review of evidence regarding clinical services commissioned from community pharmacies. Commissioned by the Chief Pharmaceutical Officer for England to inform the Murray Review of clinical services in community pharmacy. https://www.england.nhs.uk/ commissioning/wp-content/uploads/sites/12/2016/12/rapid-evdnc-rev-dec16.pdf. Accessed 6 Jan 2017.

50. Centre for Policy on Ageing - Rapid review. The effectiveness of Community Pharmacy Medication (Medicine Use) Reviews. CPA March 2014 http://www.cpa.org.uk/information/reviews/CPA-Rapid-Review-CommunityPharmacy-Medication-Reviews.pdf. Accessed Feb 2016.

51. Bagole LE, Beaumont A, Morgan I. Outcomes of medicines use reviews for people with asthma. Int J Pharm Pract. 2007;15 Suppl 2:B6.

52. Hatah E, Braund R, Tordoff J, Duffull SB. A systematic review and meta-analysis of pharmacist-led fee-for-services medication review. Br J Clin Pharmacol. 2014;77(1):102-15.

53. Legge di stabilitá, article 32 bis, medicines use review, amendment 32.0.2000, December 2015. http://www.ilfarmacistaonline.it/governo-e-parlamento/ articolo.php?articolo_id=33572. Italian. Accessed 20 Feb 2016.

\section{Submit your next manuscript to BioMed Central and we will help you at every step:}

- We accept pre-submission inquiries

- Our selector tool helps you to find the most relevant journal

- We provide round the clock customer support

- Convenient online submission

- Thorough peer review

- Inclusion in PubMed and all major indexing services

- Maximum visibility for your research

Submit your manuscript at www.biomedcentral.com/submit 